hep-ph/0001090

January 2000

Revised March 2000

\title{
Thermal Abundances of Heavy Particles
}

\author{
Mark Srednicki* \\ Department of Physics, University of California, Santa Barbara, CA 93106
}

\begin{abstract}
Matsumoto and Yoshimura hep-ph/9910393 have argued that there are loop corrections to the number density of heavy particles (in thermal equilibrium with a gas of light particles) that are not Boltzmann suppressed by a factor of $e^{-M / T}$ at temperatures $T$ well below the mass $M$ of the heavy particle. We argue, however, that their definition of the number density does not correspond to a quantity that could be measured in a realistic experiment. We consider a model where the heavy particles carry a conserved U(1) charge, and the light particles do not. The fluctuations of the net charge in a given volume then provide a measure of the total number of heavy particles in that same volume. We show that these charge fluctuations are Boltzmann suppressed (to all orders in perturbation theory). Therefore, we argue, the number density of heavy particles is also Boltzmann suppressed.
\end{abstract}

Physical Review D, in press

Typeset using REVTEX

${ }^{*}$ E-mail: mark@vulcan.physics .ucsb.edu 
In a series of papers, Matsumoto and Yoshimura (hereafter MY) [1] 3ave challenged the conventional wisdom concerning the number density of a gas of heavy particles in thermal equilibrium with a gas of light (or massless) particles. They consider a model of a heavy spin-zero boson (represented by a real scalar field $\varphi$ ) interacting with a massless spin-zero boson (represented by a real scalar filed $\chi$ ). The scalar potential is

$$
V(\varphi, \chi)=\frac{1}{2} M^{2} \varphi^{2}+\frac{1}{24} \lambda_{\varphi} \varphi^{4}+\frac{1}{24} \lambda_{\chi} \chi^{4}+\frac{1}{4} \lambda \varphi^{2} \chi^{2},
$$

where the couplings are all real and positive, and we take $\lambda_{\varphi} \ll \lambda^{2}, \lambda \ll \lambda_{\chi}$, and $\lambda_{\chi}<1$. This hierarchy among the couplings allows the $\chi$ particles to function as an efficient heat bath for the $\varphi$ particles.

At the level of free field theory, the equilibrium number density of $\varphi$ particles is given by

$$
n_{\varphi 0}=g \int \frac{d^{3} p}{(2 \pi)^{3}} f(E),
$$

where $g=1$ counts the number of species of $\varphi$ particles,

$$
f(E)=\frac{1}{e^{\beta E}-1}
$$

is the Bose distribution function, $\beta=1 / T$ is the inverse temperature, and $E=\left(\mathbf{p}^{2}+M^{2}\right)^{1 / 2}$ is the single-particle energy. For $T \ll M$, we have

$$
n_{\varphi 0}=(2 \pi)^{-3 / 2}(M T)^{3 / 2} e^{-\beta M} ;
$$

the factor of $e^{-\beta M}$ means that $n_{\varphi 0}$ is Boltzmann suppressed. However, MY argue that there are loop corrections to $n_{\varphi 0}$ that are not Boltzmann suppressed; specifically, they find [2,3]

$$
n_{\varphi}=n_{\varphi 0}+c \lambda^{2} T^{6} / M^{3}+\ldots
$$

for $T \ll M$, where $c=1 / 69120$, and the ellipses stand for all higher-order corrections.

They key issue that we wish to address (raised also in [4]) is the underlying definition of $n_{\varphi}$. For $T \ll M$, MY define $n_{\varphi}$ via $n_{\varphi}=\rho_{\varphi} / M$, where $\rho_{\varphi}$ is the energy density of the $\varphi$ particles. This energy density is in turn defined (for all temperatures) via

$$
\rho_{\varphi}=\left\langle\mathcal{H}_{\varphi}\right\rangle=\frac{\operatorname{Tr} \mathcal{H}_{\varphi} e^{-\beta H}}{\operatorname{Tr} e^{-\beta H}}-\left\langle 0\left|\mathcal{H}_{\varphi}\right| 0\right\rangle,
$$

where $H$ is the total hamiltonian, and

$$
\mathcal{H}_{\varphi}=\frac{1}{2} \dot{\varphi}^{2}+\frac{1}{2}(\nabla \varphi)^{2}+\frac{1}{2} M^{2} \varphi^{2}+\text { counterterms }
$$

is the free-field part of the $\varphi$ hamiltonian, plus counterterms (some of which involve the $\chi$ field) that are necessary to remove infinities in this composite operator.

Eq. (6) is a highly plausible definition of $\rho_{\varphi}$. However, it does not correspond in any obvious way to how the number density of $\varphi$ particles would be determined experimentally. Standard methods all involve a search for individual, on-shell $\varphi$ particles. Real-world examples of this include present-day dark matter searches, and measurements of the cosmic microwave background radiation. 
What is needed theoretically is a measurable attribute that is carried by the $\varphi$ particles only. To create one, we modify the model slightly by making the $\varphi$ field complex, and requiring its interactions to conserve the corresponding $\mathrm{U}(1)$ charge. We leave the $\chi$ field real, and the $\chi$ particles neutral. The modified scalar potential is

$$
V(\varphi, \chi)=M^{2} \varphi^{\dagger} \varphi+\frac{1}{4} \lambda_{\varphi}\left(\varphi^{\dagger} \varphi\right)^{2}+\frac{1}{24} \lambda_{\chi} \chi^{4}+\frac{1}{2} \lambda \varphi^{\dagger} \varphi \chi^{2} .
$$

We can now study the net charge contained in a large but finite volume $V$. Of course, in thermal equilibrium, the average net charge $\langle Q\rangle$ vanishes, but it has nonzero fluctuations $\left\langle Q^{2}\right\rangle$. If we weakly gauge the $\mathrm{U}(1)$ symmetry with a small (and therefore dynamically irrelevant) gauge coupling $e \ll \lambda$, we can in principle measure these charge fluctuations without tracking individual $\varphi$ particles.

For $T \ll M$, it is easy to compute $\left\langle Q^{2}\right\rangle_{0}$, where the subscript 0 indicates that we are (for now) neglecting interactions. The number $N_{+}$of positively charged particles in a volume $V$ is then controlled by a Poisson distribution; this implies $\left\langle N_{+}^{2}\right\rangle_{0}-\left\langle N_{+}\right\rangle_{0}^{2}=\left\langle N_{+}\right\rangle_{0}$. The number $N_{-}$of negatively charged particles is controlled by an independent Poisson distribution, with $\left\langle N_{-}^{2}\right\rangle_{0}-\left\langle N_{-}\right\rangle_{0}^{2}=\left\langle N_{-}\right\rangle_{0}$. Overall charge neutrality implies $\left\langle N_{-}\right\rangle_{0}=\left\langle N_{+}\right\rangle_{0}=\frac{1}{2} n_{\varphi 0} V$, where $n_{\varphi 0}$ is now given by Eq. (2) with $g=2$. The net charge is $Q=N_{+}-N_{-}$, and so

$$
\begin{aligned}
\left\langle Q^{2}\right\rangle_{0} & =\left\langle\left(N_{+}-N_{-}\right)^{2}\right\rangle_{0} \\
& =\left\langle N_{+}^{2}\right\rangle_{0}+\left\langle N_{-}^{2}\right\rangle_{0}-2\left\langle N_{+}\right\rangle_{0}\left\langle N_{-}\right\rangle_{0} \\
& =n_{\varphi 0} V .
\end{aligned}
$$

We see that the charge fluctuations give us a measurement of the total number of $\varphi$ particles in a given volume. At higher temperatures (but still ignoring interactions), quantum effects modify this result to

$$
\left\langle Q^{2}\right\rangle_{0}=2 V \int \frac{d^{3} p}{(2 \pi)^{3}} f(E)[1+f(E)] .
$$

The ratio $\left\langle Q^{2}\right\rangle_{0} / n_{\varphi 0} V$ depends weakly on temperature; it rises slowly from one at $T \ll M$ to $\pi^{2} / 6 \zeta(3)=1.37$ at $T \gg M$. (The result is similar for fermions, with a ratio of one at low temperatures and $\pi^{2} / 9 \zeta(3)=0.91$ at high temperatures.) Furthermore, it seems highly unlikely that weak interactions could significantly modify Eq. (9)). If we were to have either $\left\langle Q^{2}\right\rangle \ll n_{\varphi} V$ or $\left\langle Q^{2}\right\rangle \gg n_{\varphi} V$, we would be forced to conclude that the movements of positive and negative particles are highly correlated (in order to suppress or enhance the charge fluctuations in any particular volume). This is inconsistent with the usual notion of a gas of particles that move freely and independently between occasional scatterings, and would appear to require strong interactions.

We therefore propose to define the number density of $\varphi$ particles, for $T \ll M$, via

$$
n_{\varphi}=\left\langle Q^{2}\right\rangle / V .
$$

This definition has the advantage (not shared by the definition used by MY) of being directly connected to the experimentally measurable quantity $\left\langle Q^{2}\right\rangle$. Adopting Eq. (11) as our definition of $n_{\varphi}$, the question becomes whether or not the loop corrections to $\left\langle Q^{2}\right\rangle$ are Boltzmann suppressed. 
To compute these loop corrections, we introduce a chemical potential $\mu$ and the partition function

$$
Z=\operatorname{Tr} e^{-\beta(H-\mu Q)}
$$

We then use

$$
\left\langle Q^{2}\right\rangle=\left.\frac{1}{\beta^{2}} \frac{\partial^{2}}{\partial \mu^{2}} \ln Z\right|_{\mu=0}
$$

At the one-loop level (that is, for free $\varphi$ particles, and ignoring the $\mu$-independent contributions of the $\chi$ particles), we have the textbook formula

$$
\ln Z_{0}=V \int \frac{d^{3} p}{(2 \pi)^{3}} \ln [1+f(E-\mu)]+(\mu \rightarrow-\mu) .
$$

Using Eq. (14) in Eq. (13) yields Eq. (10).

As in [2], loop corrections to $\ln Z$ may be computed via finite temperature perturbation theory (see, e.g., [5]). At the two- and three-loop level, the contributing diagrams are shown in Fig. (1). Vertices joining four $\varphi$ lines are neglected since we have assumed that $\lambda_{\varphi} \ll \lambda^{2}$. Furthermore we neglect diagrams with only $\chi$ lines, since these are independent of $\mu$. The $\varphi$ propagator is

$$
\Delta(n, \mathbf{p})=\frac{1}{(2 \pi n / \beta-i \mu)^{2}+\mathbf{p}^{2}+M^{2}},
$$

where $n$ is an integer specifying the discrete energy. (With $M=\mu=0$, this is also the $\chi$ propagator.) The direction of the flow of charge is the same as the flow of energy. A closed $\varphi$ loop with a single vertex contributes a factor of

$$
T \sum_{n=-\infty}^{+\infty} \Delta(n, \mathbf{p})=\frac{1}{2 E}[1+f(E+\mu)+f(E-\mu)],
$$

where $E=\left(\mathbf{p}^{2}+M^{2}\right)^{1 / 2}$. The "1" in square brackets yields a divergent, temperature independent term that must be removed by renormalization. The remaining two terms are Boltzmann suppressed, since at low temperatures

$$
f(E \pm \mu) \simeq e^{-\beta M} e^{\mp \beta \mu} e^{-\beta \mathbf{p}^{2} / 2 M} .
$$

This implies that the two-loop diagram in Fig. (1), and the first two three-loop diagrams, are all Boltzmann suppressed. The third three-loop diagram has a factor of $\sum_{n} \Delta(n, \mathbf{p})^{2}$, and turns out to be suppressed as well. This leaves only the last ("basketball") diagram.

According to the standard rules of finite temperature perturbation theory [5], the contribution of the basketball diagram is

$$
\begin{aligned}
\frac{1}{\beta V} \ln Z_{\mathrm{bb}}= & C \lambda^{2} T^{4} \sum_{n^{\prime} \mathrm{s}} \int \frac{d^{3} p_{1}}{(2 \pi)^{3}} \frac{d^{3} p_{2}}{(2 \pi)^{3}} \frac{d^{3} k_{3}}{(2 \pi)^{3}} \frac{d^{3} k_{4}}{(2 \pi)^{3}}(2 \pi)^{3} \delta^{3}\left(\mathbf{p}_{1}+\mathbf{p}_{2}+\mathbf{k}_{3}+\mathbf{k}_{\mathbf{4}}\right) \\
& \times \beta \delta_{n_{1}+n_{2}+n_{3}+n_{4}, 0} \Delta_{1} \Delta_{2} \Delta_{3} \Delta_{4} .
\end{aligned}
$$



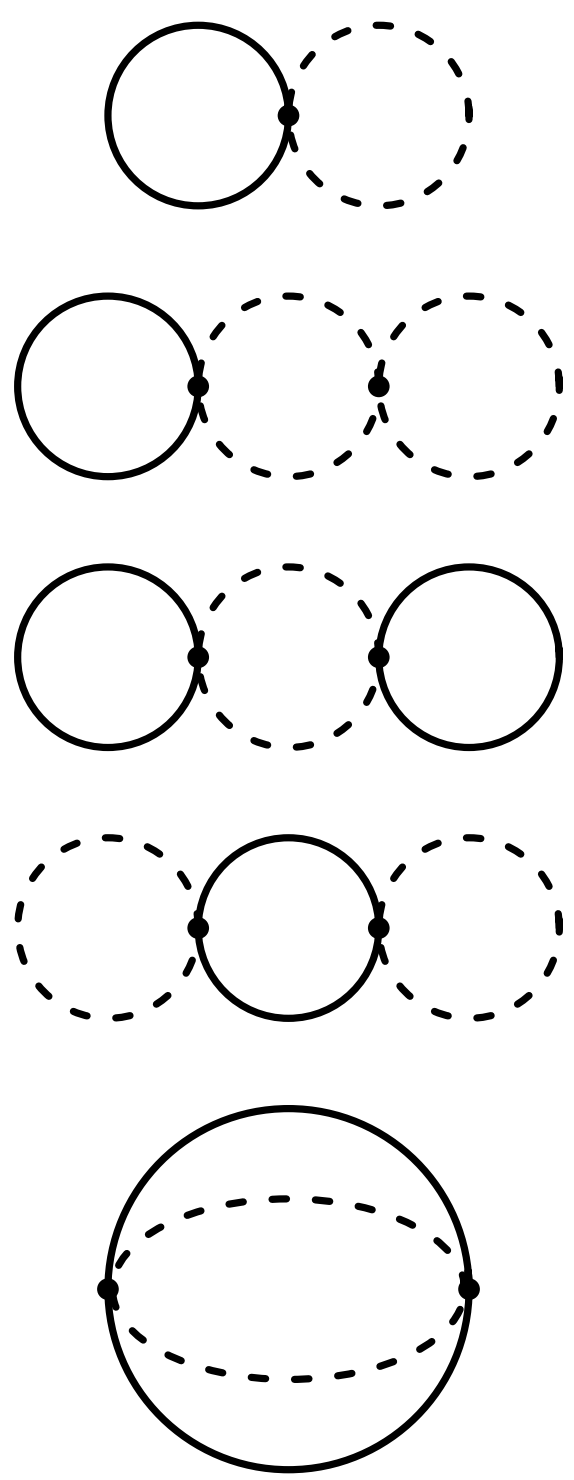

FIG. 1. Two- and three-loop contributions to $\ln Z / \beta V$ that include propagators for both the heavy $\varphi$ particle (solid lines) and the massless $\chi$ particle (dashed lines). 
Here $\mathbf{p}_{1}$ and $\mathbf{p}_{2}$ are the momenta on the $\varphi$ lines, and $\mathbf{k}_{3}$ and $\mathbf{k}_{4}$ are the momenta on the $\chi$ lines. Each momentum and energy is taken to flow from the left vertex to the right vertex. Thus we have, in the individual propagators, masses $M_{1}=M_{2}=M$ and $M_{3}=M_{4}=0$, and chemical potentials $\mu_{1}=-\mu_{2}=\mu$ and $\mu_{3}=\mu_{4}=0$. Note especially that $\mu_{2}=-\mu_{1}$; this is because, with our convention on energy flow, the charge flow must be opposite to the energy flow on one of the $\varphi$ lines, and this changes the sign of the chemical potential. Finally,

$$
C=\frac{1}{2 !} \cdot\left(-\frac{1}{2}\right)^{2} \cdot 2=\frac{1}{4}
$$

is a combinatoric factor. The $1 / 2$ ! comes from the expansion of $\exp \left(-\beta H_{\mathrm{int}}\right)$, the $(-1 / 2)^{2}$ comes from two vertex factors arising from the last term in the scalar potential, Eq. (8), and the 2 comes from the number of ways to match up the $\chi$ lines.

Eq. (18) can be evaluated by the standard procedure [5] of writing the Kroneker delta as an integral,

$$
\beta \delta_{n_{1}+\ldots+n_{4}, 0}=\int_{0}^{\beta} d t e^{2 \pi i\left(n_{1}+\ldots+n_{4}\right) t / \beta},
$$

and then performing each sum via contour integration,

$$
\begin{aligned}
\sum_{n=-\infty}^{+\infty} \frac{e^{2 \pi i n t / \beta}}{(2 \pi n / \beta-i \mu)^{2}+E^{2}} & =\frac{\beta}{2 \pi} \oint d z \frac{e^{i z t}}{e^{i \beta z}-1} \frac{1}{(z-i \mu)^{2}+E^{2}} \\
& =\frac{\beta}{2 E}\left[e^{(\beta-t)(E+\mu)} f(E+\mu)+e^{t(E-\mu)} f(E-\mu)\right] .
\end{aligned}
$$

Here the $z$ contour originally encloses the real axis, and then is deformed into two small circles surrounding the poles at $z=i(\mu \pm E)$; this deformation is allowed for $0 \leq t \leq \beta$. Putting all of this together we now have

$$
\frac{1}{\beta V} \ln Z_{\mathrm{bb}}=C \lambda^{2} \int \widetilde{d p}_{1} \widetilde{d p}_{2} \widetilde{d k}_{3} \widetilde{d k}_{4}(2 \pi)^{3} \delta^{3}\left(\mathbf{p}_{1}+\mathbf{p}_{2}+\mathbf{k}_{3}+\mathbf{k}_{\mathbf{4}}\right) \int_{0}^{\beta} d t D_{1} D_{2} D_{3} D_{4},
$$

where $\widetilde{d p}_{i}=d^{3} p_{i} /(2 \pi)^{3}\left(2 E_{i}\right)$, and

$$
D_{i}=\left[e^{(\beta-t)\left(E_{i}+\mu_{i}\right)} f\left(E_{i}+\mu_{i}\right)+e^{t\left(E_{i}-\mu_{i}\right)} f\left(E_{i}-\mu_{i}\right)\right] .
$$

The integral over $t$ is tedious but straightforward to perform; the result is

$$
\begin{aligned}
\int_{0}^{\beta} d t D_{1} D_{2} D_{3} D_{4}= & \sum_{\delta_{1}=0}^{1} \sum_{\delta_{3}=0}^{1} \sum_{\delta_{4}=0}^{1}\left[\frac{\left(\delta_{1}+f_{1+}\right)\left(\delta_{1}+f_{2-}\right)\left(\delta_{3}+f_{3}\right)\left(\delta_{4}+f_{4}\right)}{\varepsilon_{1} E_{1}+\varepsilon_{1} E_{2}+\varepsilon_{3} E_{3}+\varepsilon_{4} E_{4}}\right. \\
& \left.+\frac{\left(\delta_{1}+f_{1+}\right)\left(1-\delta_{1}+f_{2+}\right)\left(\delta_{3}+f_{3}\right)\left(\delta_{4}+f_{4}\right)}{\varepsilon_{1} E_{1}-\varepsilon_{1} E_{2}+\varepsilon_{3} E_{3}+\varepsilon_{4} E_{4}}\right]+(\mu \rightarrow-\mu),
\end{aligned}
$$

where $\varepsilon_{i}=(-1)^{1-\delta_{i}}$, and $f_{1+}=f\left(E_{1}+\mu\right), f_{2-}=f\left(E_{2}-\mu\right), f_{3}=f\left(E_{3}\right)$, etc. We have made repeated use of the relation

$$
e^{\beta E} f(E)=1+f(E)
$$


in obtaining Eq. (24).

We are interested only in those terms (if any) in Eq. (24) that are not Boltzmann suppressed. As we see from Eq. (17), any term containing a factor of either $f_{1 \pm}$ or $f_{2 \pm}$ is Boltzmann suppressed. Thus, we can drop all such terms. At this point, we see immediately that the remaining terms are all independent of $\mu$, since the only $\mu$ dependence in Eq. (24) is that which is contained implicitly in the factors of $f_{1 \pm}$ and $f_{2 \pm}$. Therefore, all $\mu$-dependent contributions (at the two- and three-loop level) to $\ln Z$ are Boltzmann suppressed. Eqs. (13) and (11) then imply that $\left\langle Q^{2}\right\rangle$ and $n_{\varphi}$ are also Boltzmann suppressed. This is our main result.

For completeness, and for comparison with the results of MY [2], we continue the computation of the unsuppressed terms in $\ln Z_{\mathrm{bb}}$. We set $f_{1 \pm}=f_{2 \pm}=0$. All numerators in Eq. (24) are then either $1, f_{3}, f_{4}$, or $f_{3} f_{4}$. The first of these yields a divergent, temperature independent term that is canceled by a renormalization of the vacuum energy. The second and third yield divergent, temperature dependent terms that are canceled by renormalization of the $\varphi$ and $\chi$ thermal self-energies (see the corresponding discussion for QED in [5]). Dropping these terms, we have

$$
\int_{0}^{\beta} d t D_{1} D_{2} D_{3} D_{4} \rightarrow \sum_{\varepsilon_{3}= \pm 1} \sum_{\varepsilon_{4}= \pm 1} \frac{2 f_{3} f_{4}}{E_{1}+E_{2}+\varepsilon_{3} E_{3}+\varepsilon_{4} E_{4}}
$$

Substituting this into Eq. (22), making a low temperature expansion, and dropping a final divergent contribution to the vacuum energy ultimately yields

$$
\frac{1}{\beta V} \ln Z_{\mathrm{bb}}=\frac{\pi^{2} \lambda^{2} T^{8}}{648000 M^{4}}+O\left(T^{10}\right)
$$

The corresponding correction to the total energy density $\rho$ is then given by

$$
\delta \rho=-\frac{1}{V} \frac{\partial}{\partial \beta} \ln Z_{\mathrm{bb}}=\frac{7 \pi^{2} \lambda^{2} T^{8}}{648000 M^{4}}+O\left(T^{10}\right) .
$$

This is consistent with the results of MY [2], who also find that the leading correction to $\rho$ is of order $T^{8} / M^{4}$.

That there are corrections of this form to $\rho$ is not surprising. Without interactions, we have $\rho=\left(\pi^{2} / 30\right) T^{4}$ from the $\chi$ particles, plus the Boltzmann-suppressed contribution of the $\varphi$ particles. At temperatures $T \ll M$, we should be able to integrate the heavy $\varphi$ field out of the functional integral, and be left with an effective lagrangian for the massless $\chi$ field alone. This lagrangian will contain nonrenormalizable interaction terms that are suppressed by powers of $\lambda$ and inverse powers of $M$. These will give rise to corrections like Eq. (28). From this point of view, it is clear that all such corrections should be thought of as modifications of the energy of the $\chi$ particles, and not as unsuppressed contributions to the energy of the $\varphi$ particles.

We now argue that $\left\langle Q^{2}\right\rangle$ (and hence $n_{\varphi}$ ) is Boltzmann suppressed to all orders in perturbation theory. Consider an exact evaluation of the partition function

$$
Z=\sum_{\alpha} e^{-\beta\left(E_{\alpha}-\mu Q_{\alpha}\right)}
$$


where the sum is over a basis of energy and charge eigenstates. States that yield a $\mu$ dependent contribution to $Z$ must have $Q \neq 0$. States consisting of a single stable $\varphi$ particle are well defined exact energy eigenstates, with $E=\left(\mathbf{p}^{2}+M^{2}\right)^{1 / 2}$ and $Q= \pm 1$; their contribution to $Z$ is obviously Boltzmann suppressed. Furthermore, any other state with $Q= \pm 1$ must have energy $E>M$; otherwise, the $\varphi$ particle would not be stable (since it could decay into this lighter charged state). At tree level, energy eigenstates with two or more $\varphi$ particles all have $E \geq 2 M$; interactions can modify this to $E \geq 2 M\left[1+O\left(\lambda^{2}\right)\right]$. The $O\left(\lambda^{2}\right)$ term could in principle be negative (if there is a bound state of two or more like-charge particles), but cannot make $E \ll 2 M$ in a weakly coupled theory. We therefore conclude that all $\mu$-dependent contributions to $Z$ are Boltzmann suppressed. We have already seen this explicitly at the level of two and three loops.

With this in mind, we can return to the original model of MY, Eq. (1). At the level of Feynman diagrams, the models are essentially identical, the only difference being the combinatoric factor associated with each diagram. We therefore expect that $n_{\varphi}$ should be Boltzmann suppressed in this model as well. We are still in need, however, of a general definition of $n_{\varphi}$ that does not rely on the trick of Eq. (11), but which corresponds to experimental measurements.

We conclude, in accord with [4], that the proper definition of the number density $n_{\varphi}$ of heavy particles (in equilibrium with a thermal bath of light particles) is a delicate matter. We have argued that it is essential that $n_{\varphi}$ be defined in a manner that renders it measurable in a realistic experiment. In this paper, we have considered a model in which the heavy particles carry a conserved $\mathrm{U}(1)$ charge, while the light particles are neutral. In this situation, the local charge fluctuations provide an experimentally accessible measure of the number density of heavy particles. We have shown explicitly that, at low temperatures, this number density is Boltzmann suppressed up through three-loop order in perturbation theory, and we have argued that this must in fact be true to all orders.

\section{ACKNOWLEDGMENTS}

I thank Anupam Singh and Scott Thomas for discussions. This work was supported in part by the National Science Foundation through grant PHY-97-22022, and by the Institute of Geophysics and Planetary Physics through grant 920. 


\section{REFERENCES}

[1] Sh. Matsumoto and M. Yoshimura, Phys. Rev. D 59, 123511 (1999), hep-ph/9811301.

[2] Sh. Matsumoto and M. Yoshimura, hep-ph/9910393.

[3] Sh. Matsumoto and M. Yoshimura, hep-ph/9910425.

[4] A. Singh and M. Srednicki, Phys. Rev. D 61, 023509 (2000), hep-ph/9908224.

[5] J. I. Kapusta, Finite-temperature field theory (Cambridge, 1989). 